\section{All four dengue virus serotypes co-circulate in concurrent dengue infections in a single dengue session in Chittagong, Bangladesh}

\author{
Moushumi Ghosh Roy', 2, Kutub Uddin³, Din Islam², Anjuvan Singh4 and \\ Mohammad Monirul Islam ${ }^{2 \star}$
}

\author{
${ }^{1}$ Department of Biochemistry, Lovely Professional University, Punjab, India \\ ${ }^{2}$ Department of Biochemistry and Molecular Biology, University of Chittagong, Chittagong 4331, \\ Bangladesh \\ ${ }^{3}$ Department of Medicine, Chittagong Medical College Hospital, Chittagong, Bangladesh \\ ${ }^{4}$ Department of Biotechnology, School of Bioengineering and Bioscience, Lovely Professional \\ University, Punjab, India
}

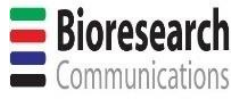

Bioresearch Communications

Volume 8, Issue 1, January 2022

DOI:

https://doi.org/10.3329/brc.v8i1.57042

\begin{abstract}
Purposes: Dengue fever, a mosquito-borne viral disease, is a global public health burden affecting millions of people each year and over $40 \%$ of world populations are at risk of dengue. Therefore, prompt and accurate dengue diagnosis is inevitable for disease surveillance and for aiding disease management. In this study we report dengue virus (DENV) seroprevalence in Chittagong, Bangladesh along with clinical manifestation of dengue infections. Methods: All samples included in this study were selected based on dengue NS1-based diagnosis, clinical sign and symptoms were judged by expert clinical physicians and infecting DENV serotyping was done by RT-PCR. The blood cells (Platelet, Haematocrit, WBC etc) were analyzed using Haematology cell counter. Results: First, among the 112 DENV infected serum samples tested by RT-PCR, 42 were DENV positive where $76 \%$ samples had single DENV serotype infection and $24 \%$ were concurrently infected with two or more DENV serotypes, indicating that all four DENVs were present in a single dengue session in Chittagong, Bangladesh. Then, DENV4 was the most prevailed serotype, followed by DENV2, DENV1 and DENV3 in single DENV serotype infections. However, in almost $90 \%$ cases of concurrent multiple DENV infections DENV1 serotype was present. A detail analysis of clinical data clearly indicated that DENV1 and DENV2 resulted very similar patterns of clinical symptoms which were quite different from those caused by DENV3 and DENV4. For example, ache and pain were absent in DENV3 infection and diarrhea was absent in DENV4 infections. Furthermore, DENV3, both in single and concurrent multiple DENV infections, might increase dengue disease severity as observed highly reduced platelet counts along with increased WBC in patients infected with DENV3 serotype. Conclusion: All four DENV serotypes, both as single and concurrent multiple DENV infections, were present in single dengue session in Bangladesh. Despite having very similar sequences and structures all four DENVs might produce different disease spectra, ranging from classical dengue fever to dengue hemorrhagic fever. Concurrent multiple DENV infections could contribute increased dengue disease severity in dengue outbreaks in Bangladesh.
\end{abstract}

KEYWORDS: Dengue infection; Dengue Seroprevalence; Disease Severity; Co-circulation of DENVs; Concurrent DENV infections.

RECEIVED: 09 August 2021, ACCEPTED: 16 October 2021

TYPE: Original Research
CORRESPONDING AUTHOR: Mohammad Monirul Islam, Department of Biochemistry and Molecular Biology, University of Chittagong, Chittagong 4331, Bangladesh. Email: islammm@cu.ac.bd

\section{Introduction}

Dengue is a mosquito-borne viral disease in tropical and subtropical countries and is caused by structurally similar but antigenically distinct four dengue virus serotypes (DENV1 4) (Stanaway et al., 2016; WHO, 2016; Bhatt et al., 2013; Guzman et al., 2010; Halstead, 1966). Human is the only known host for DENVs and DENV infections cause various degrees of disease spectrum, ranging from undifferentiated fever to severe hemorrhagic fever. Although a considerable proportion of persons being infected with DENVs remain asymptomatic, the dengue is preliminary characterized by typical high fever, named as "dengue fever (DF)", and in severe cases, DENV infections manifest dengue hemorrhagic fever (DHF) and dengue shock syndrome (DSS) (Soo et al., 2016). The clinical symptoms of dengue fever, in general, are high fever, headache, myalgia, arthragia, and rash. On the other hand, symptoms of dengue hemorrhagic fever and dengue shock syndrome include hemorrhagic manifestation, leakage of vascular fluids and shock accompanied by thrombocytopenia and abundant complement activation (Guzman et al., 2013). The DHF and DSS are the major complications of severe dengue manifestation and at these stages of dengue infection, immediate hospitalization is required. Though the DHF was first demonstrated in Southeast Asia almost over 50 years ago, now a days it is quite seldom observed in other countries in recent dengue outbreaks (Gubler, 1998; Nimmannitya et al., 1987).

Any DENV serotype infection can produce full spectrum of illnesses and severity, ranging from non-specific febrile syndrome, flu-like fever and classical DF to DHF and DSS (Nimmannitya et al., 1987). In addition, almost half of DENV infected individuals are either asymptomatic or have an undifferentiated fever (Ten et al., 2018). Consequently, dengue diagnosis based on clinical syndromes is not reliable and there is no dengue-specific treatment, together affecting 
dengue disease management (Chastel, 2012; Duong et al., 2015; Senaratne et al., 2020). Several laboratory-based dengue diagnostic methods detecting dengue virus, viral RNA (Lanciotti et al.,1992) viral antigens (Matheus et al., 2005)_and anti-dengue antibodies have been reported (Kuno et al., 1991; Innis et al., 1989). At present, the non-structural protein 1 (NS1) based diagnosis and MAC-ELISA are quite commonly used (Crooks et al., 1990; Pok et al., 2010). However, the NS1 is very short-lived in infected human blood and it suffers from high false negative prediction (Pok et al., 2010; Acosta et al., 2014). Moreover, much details on infecting DENV serotypes and its association with clinical dengue manifestation in Bangladesh is yet to be available. Therefore, in this study we attempted to explore DENV seroprevalence along with DENV serotype-specific clinical manifestations of dengue infection in Chittagong, Bangladesh.

\section{Materials and Methods}

\section{Collection of DENV infected human sera}

This study was performed with a total of 112 DENV infected serum specimens obtained from Chittagong Medical College Hospital (following ethical approval from Ethical Review Committee of Chittagong Medical College and Hospital and written consent of the participants). Among the study subjects, $78.57 \%$ and $21.42 \%$ were male and female, respectively in the age group $19-50$ years where $40.47 \%$, and $59.52 \%$ subjects had been living in urban and rural areas, respectively. The serum specimens were collected from confirmed dengue infected patients (DENV NS1 positive; July-August 2019) admitted in the hospital for treatment purposes. All serum samples were heat inactivated and preserved as $100 \mu \mathrm{L}$ aliquots at $-80^{\circ} \mathrm{C}$ until used.

\section{DENV serotyping}

First total RNAs were extracted and purified from $100 \mu \mathrm{L}$ of serum samples using RNA extraction kit (Promega Corporation). The cDNAs were synthesized using DENVspecific primers (Yong et al., 2007). To be more precise, the reaction mixture $(7.8 \mu \mathrm{L}$ of RNA extract, $1 \mu \mathrm{L}$ Oligo dT, $1 \mu \mathrm{L}$ random primer and $0.8 \mu \mathrm{L}$ DENV conserved primer) was heated at $70^{\circ} \mathrm{C}$ for $5 \mathrm{~min}$, followed by immediate cooling on ice. Then PCR master mix $(9.4 \mu \mathrm{L})$ was added and PCR was done using thermal cycler (Thermo Fisher Scientific, USA; at $25^{\circ} \mathrm{C}$ for $5 \mathrm{~min}, 42^{\circ} \mathrm{C}$ for $60 \mathrm{~min}$, and $70^{\circ} \mathrm{C}$ for $15 \mathrm{~min}$ ). Finally, infecting DENV serotypes were confirmed using DENV serotype-specific primers (DENV1 $=5$-CCC-CGT-AACACT-TTG-ATC-GCT-CCA-TT-3; DENV2 = 5'CGC-CACAAG-GGC-CAT-GAA-CAG-3', DENV3=5'-GCA-CAT-GTTGAT-TCC-AGA-GGC-TGT-C-3'; DENV4=5'-GTT-TCCAAT-CCC-ATT-CCT-GAA-TGT-GGT-GT-3') following universal cyber-green PCR protocol [cDNA $(4 \mu \mathrm{L})$; Primers (DENV-specific and DENV conserved $0.5 \mu \mathrm{L}+0.5 \mu \mathrm{L}$ ); and master mix $(10 \mu \mathrm{L}) ; \mathrm{H}_{2} \mathrm{O}(5 \mu \mathrm{L})$. The PCR protocol was as step 1: $96^{\circ} \mathrm{C}$ for $2 \mathrm{~min}$; step 2: $96^{\circ} \mathrm{C}$ for $15 \mathrm{sec}$; step $3: 55^{\circ} \mathrm{C}$ for $33 \mathrm{sec}$; step 4: $72^{\circ} \mathrm{C}$ for $1 \mathrm{~min}$. Steps $2-4$ were repeated for 45 times and fluorescence data were recorded at $72^{\circ} \mathrm{C}$ ] using qTower (Analyticazena). Furthermore, the reconfirmation of infecting DENV serotypes was done using a single step qRTPCR dengue serotyping kit (Fast Track Dengue Diagnostics).

\section{Clinical manifestation of dengue infection}

Fever, vomiting, musculoskeletal pain, myalgia, retro-orbitalpain, rash, nausea, headache, ache-pain, diarrhea, photophobia and jaundice have been considered as clinical sign and symptoms of dengue infections and recorded by expert clinical physicians at Chittagong Medical College Hospital, Chittagong, Bangladesh. Other clinical investigations (determination of Platelet, Haematocrit, WBC etc.) following dengue infections were done using Haematology cell counter (Mindray BC- 5150) (Minnie et al., 2012).

\section{Results and Discussion \\ DENV Seroprevalence in Chittagong}

DENV serotyping by RT-PCR indicated that 42 out of 112 were DENV positive in which 32 (76\%) samples were infected with single DENV serotype and 10 (24\%) samples were found concurrently infected with two or more DENV serotypes (Figure 1). Among the single DENV serotype infections, DENV4 was the most prevailed serotype, followed by DENV2, DENV1 and DENV3 serotypes which was in contrast to what had been reported earlier in Bangladesh (Shirin et al.,2019). To be more precise, percentage of DENV4, DENV2, DENV1 and DENV3 infections were 41, 25, 22 and 12, respectively (Figure 1). 

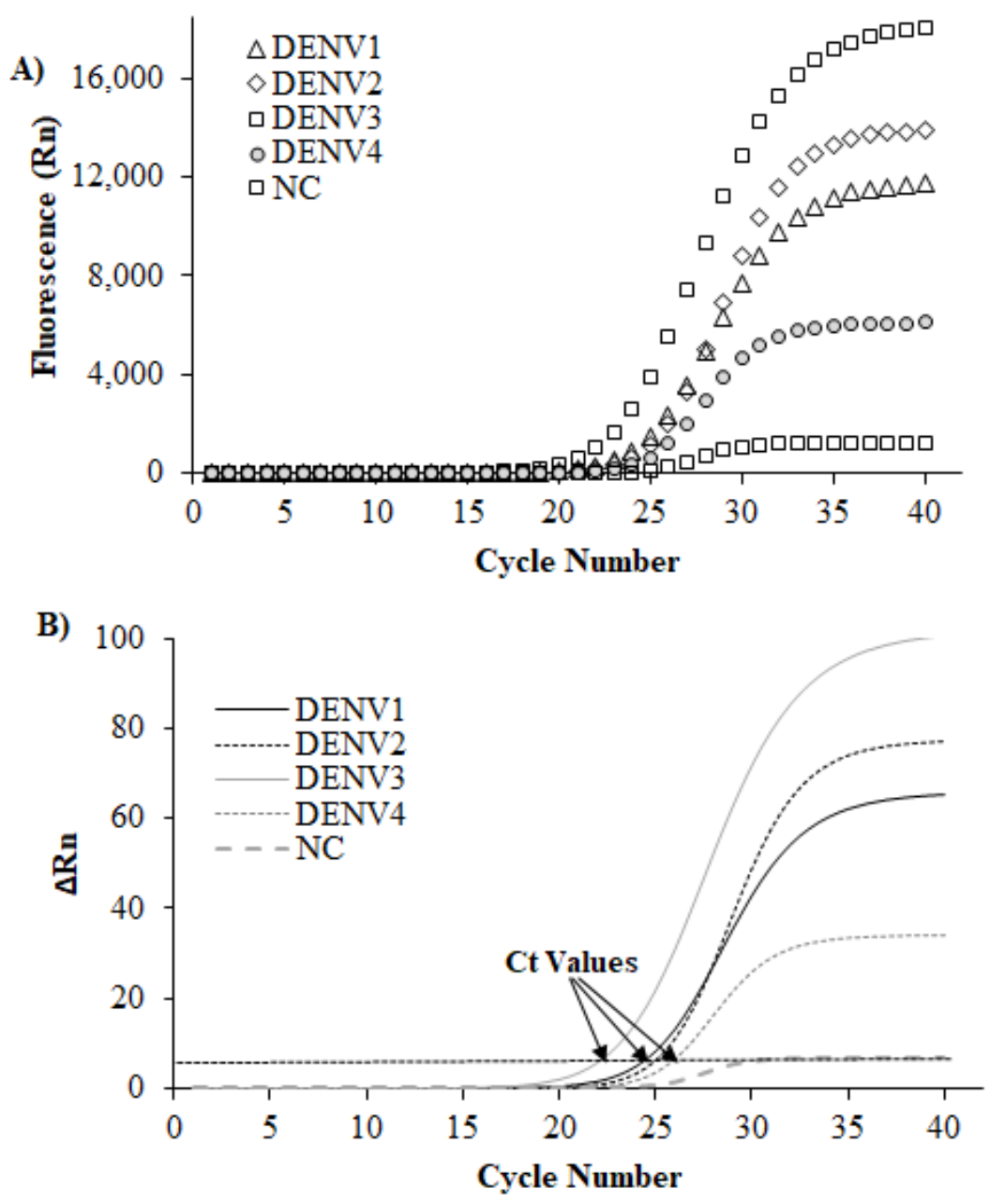

C)

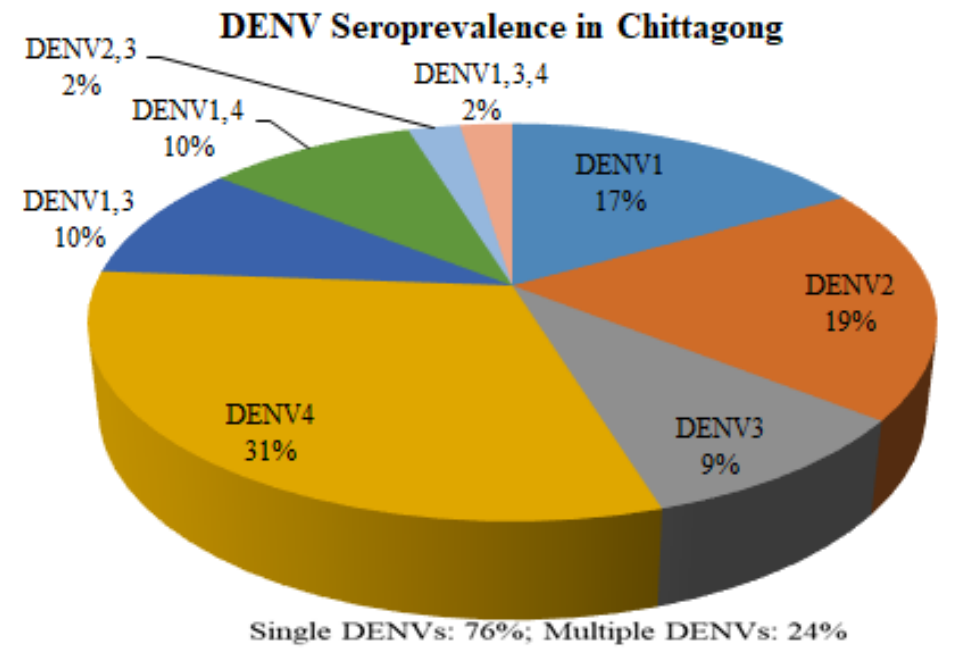

Figure 1. DENV Seroprevalence in Chittagong, Bangladesh. Serotyping was done by RT-PCR. RT-PCR normalized and fitted data are shown in panels A and B, respectively. DENV seroprevalence in Chittagong, Bangladesh is shown in panel C. Among 112 samples tested 42 were positive for DENV serotypes. Relative percentage of DENV serotypes are shown within the figure. $76 \%$ samples were infected with single DENV serotype while remaining $24 \%$ were concurrently infected with multiple DENV serotypes. 
However, several previous studies showed that the most prevalent serotype was DENV3 in India (Mehta et al., 2018) and Bangladesh (Shirin et al., 2019), indicating a seasonal and/or geolocational variations in infecting DENV serotypes from year to year which might have been contributing to increased dengue incidences and disease severity in recent years.

Interestingly, 9 out of 10 samples (90\%) being concurrently infected with multiple DENV serotypes, had DENV1 serotype in common, indicating that DENV1 was the most prevailed infecting DENV serotype in Chittagong, followed by DENV3, DENV4 and DENV2 in concurrent multiple DENV infections (Figure 1), very similar to what had been reported worldwide in recent dengue outbreaks (Masyeni et al., 2019; Tazeen et al., 2017; Utama et al., 2019; Reddy et al., 2017) Though at present we do not have any definitive explanation for such observations, however, we anticipate that natural immune response against DENV1 would be cross-reacting and it has been observed that immune responses against DENV1 generated DENV1-DENV2-DENV3 cross-reacting IgG both in mice models and natural DENV infections (personal communication), allowing other DENV serotype infections, either as heterotypic secondary and/or as concurrent multiple DENV infections (Dhanoa et al., 2016; Thomas et al., 2008). Further studies using artificial immunization and detection of natural anti-dengue antibodies may explore the facts lying behind such high DENV1 incidence in multiple DENV infections.

\section{Serotype-Specific Clinical Manifestation of DENV Infections}

Regardless of the infecting DENV serotypes, the clinical sign and symptoms including fever, vomiting, musculoskeletal pain, myalgia, re-o-pain, rash, nausea and headache were observed in almost all cases of DENV infections (Table 1).

Table 1. Clinical outcome of Dengue infection

\begin{tabular}{|l|c|c|c|c|c|c|c|c|c|c|}
\hline $\begin{array}{l}\text { Serotype } \\
\text { Number, } \boldsymbol{n})\end{array}$ & Fever & vomiting & M-pain & $\begin{array}{c}\text { Ache \& } \\
\text { Pain }\end{array}$ & Myalgia & $\begin{array}{c}\text { Re-o- } \\
\text { pain }\end{array}$ & Rash & Diarrhea & Nausea & Headache \\
\hline DENV1 $(n=7)$ & $100 \%$ & $42 \%$ & $42 \%$ & $42 \%$ & $42 \%$ & $28 \%$ & $14 \%$ & $14 \%$ & $28 \%$ & $28 \%$ \\
\hline DENV2 $(n=8)$ & $100 \%$ & $25 \%$ & $25 \%$ & $12 \%$ & $50 \%$ & $37 \%$ & $25 \%$ & $12 \%$ & $25 \%$ & $62 \%$ \\
\hline DENV3 $(n=4)$ & $100 \%$ & $25 \%$ & $50 \%$ & -- & $100 \%$ & $50 \%$ & $50 \%$ & $25 \%$ & $25 \%$ & $100 \%$ \\
\hline DENV4 $(n=13)$ & $100 \%$ & $30 \%$ & $23 \%$ & $38 \%$ & $84 \%$ & $30 \%$ & $15 \%$ & -- & $53 \%$ & $100 \%$ \\
\hline DENV1,4 $(n=4)$ & $100 \%$ & $66 \%$ & $33 \%$ & $100 \%$ & $66.66 \%$ & $66 \%$ & - & - & $100 \%$ & $100 \%$ \\
\hline DENV1,3 $(n=4)$ & $100 \%$ & $66 \%$ & & $16 \%$ & $66 \%$ & $33 \%$ & $16 \%$ & $16 \%$ & $83 \%$ & $100 \%$ \\
\hline DENV1,3,4 $(n=1)$ & $100 \%$ & $100 \%$ & & & $100 \%$ & & & & $100 \%$ & $100 \%$ \\
\hline
\end{tabular}

A detail analysis of clinical data clearly indicated that DENV1 and DENV2 resulted very similar patterns of clinical symptoms which were quite different from those caused by DENV3 and DENV4. However, ache-pain and diarrhea were absent in DENV3 and DENV4 infections, respectively. Although the number of samples analyzed in each category were very limited, it might be, at least, partially possible to assign the infecting DENV serotypes based on the clinical sign and symptoms. Moreover, based on the appearance of clinical sign and symptoms dengue treatment can be started before going through the lengthy and costly diagnosis procedure. Further studies with increased number of samples are needed for the development of the rationale between infecting DENV serotype and clinical dengue manifestation.

\section{Clinical Outcomes of DENV Infections}

Clinical dengue manifestation starts with virus replication in the infected bodies followed by cytokine release increasing vascular permeability, plasma leakage, decreasing platelet and leucocyte counts and increasing RBC in circulation (Singh et al., 2020). In all the 42 RT-PCR positive samples, platelet counts dropped significantly on day 3-4 of infection at varying degrees depending on the infecting DENV serotypes (Figure 2a). To be more precise, in the cases of DENV1, DENV2 and DENV4 infections, decrease in platelet counts was a bit slower and platelet counts started increasing within short period (after day 3). On the other hand, in the cases of single serotypic DENV3 infections, platelet counts dropped very sharply (started after day 0) and platelet counts continued decreasing till day 4 (Figure 2a). Moreover, decrease in platelet counts continued further if infected sera had DENV3 along with any other DENV serotypes in concurrent multiple DENV infections (Figure 2a). 

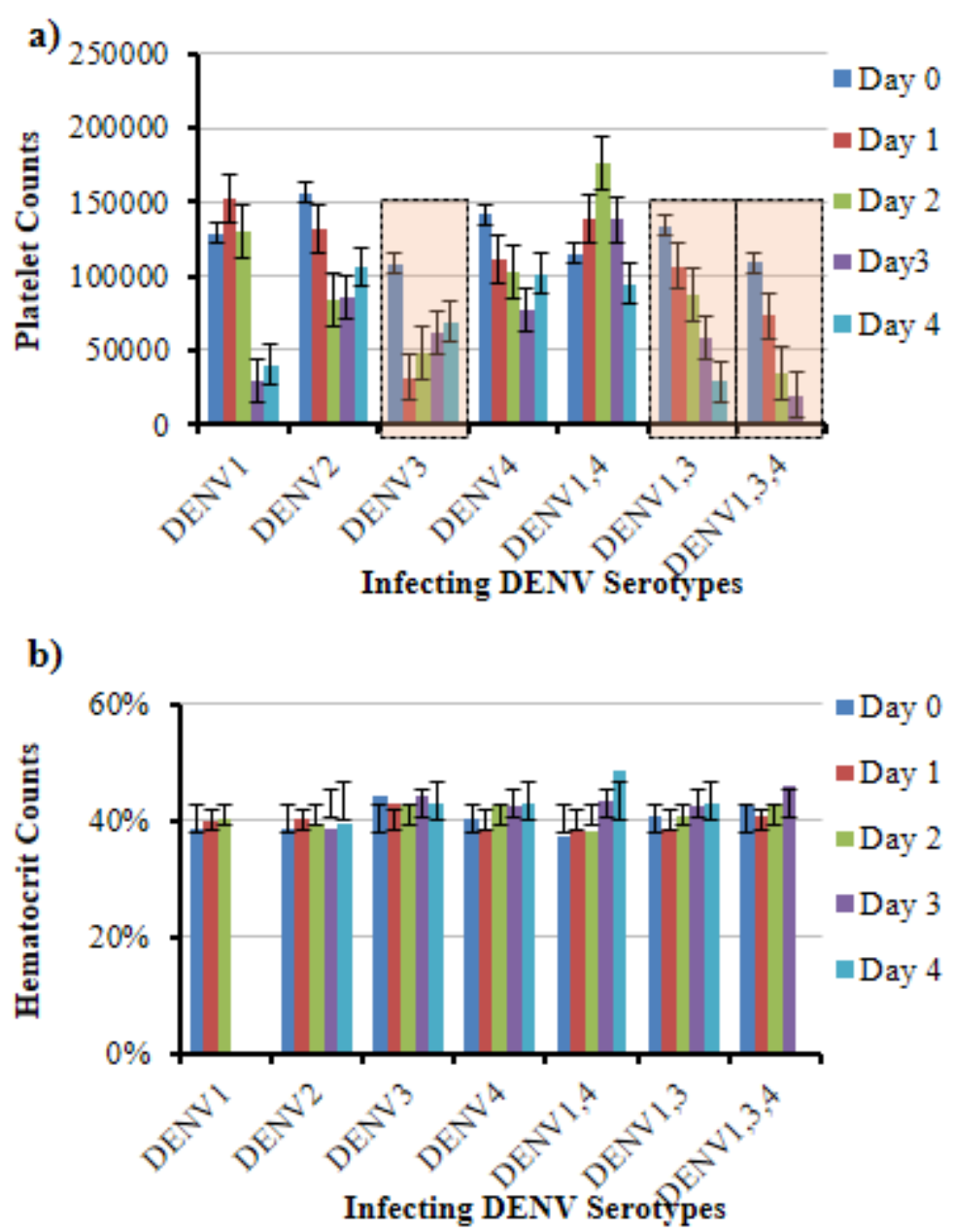

\section{c)}

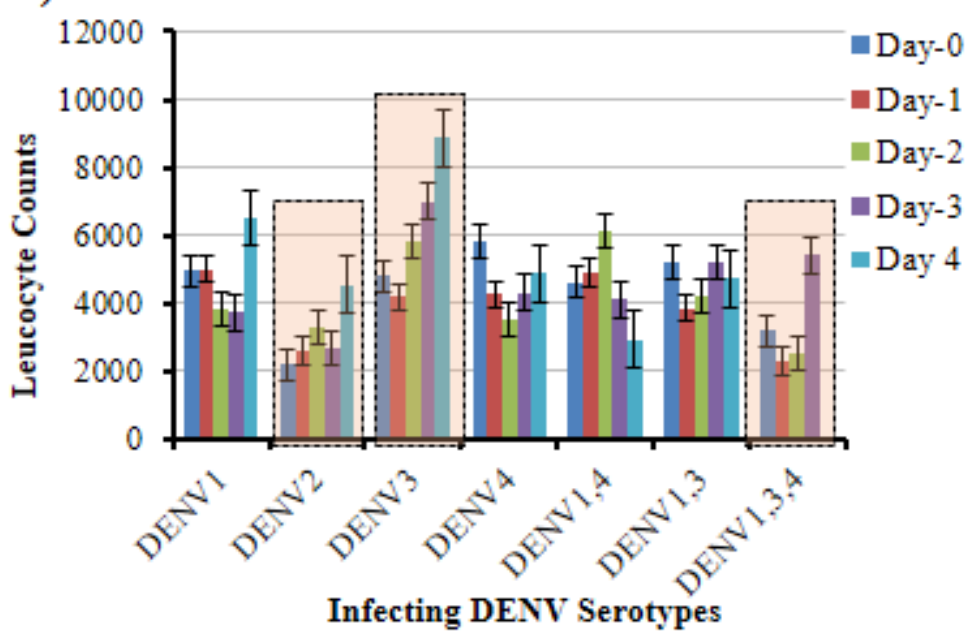

Figure 2. Clinical manifestation of dengue versus infecting DENV serotypes. Effects of infecting DENV serotypes on (a) platelet counts (per microliter of blood) (b) hematocrit counts (\%) and (c) leucocyte counts (per microliter of blood) are shown. Legends are the same for all panels and are shown at the right side of each panel. Platelet counts started decreasing soon after DENV infections and continued decreasing when the patients were infected with DENV3 along with any other DENV serotypes in concurrent multiple DENV serotype infections. For all cases the hematocrit values remained almost unaffected. However, leukocyte count remained very low following DENV2 and DENV1,3,4 infection. 
These results clearly indicated that DENV3, both as a single serotype and in concurrent multiple DENV infection might be the most fetal DENV serotype resulting thrombocytopenia, plasma leakage, dengue hemorrhagic fever and dengue shock syndrome. Severe thrombocytopenia $\left(<100,000 / \mathrm{mm}^{3}\right)$ has also been reported to be associated with overt plasma leakage contributing to dengue severity (WHO National guideline 2018).

On the other hand, regardless of the infecting DENV serotypes, the hematocrit value (RBC) (Figure 2b) and leucocyte counts (WBC) remained almost unaffected, except that WBC counts increased to some extent on the day 4 following DENV3 infections (Figure 2c) resulting leukocytosis. Moreover leucocyte count remained very low for day 0-3 following DENV2 infections and for day 0-2 following concurrent DENV1,3,4 infections showing leucopenia. Leukopenia with $<5000$ cells $/ \mathrm{mL}$ is an indication for entering into the critical phase (Figure 2c) of infection and suggested that DENV2 infected patients might experience increased severity as well (Mollah et al., 2020). However no definite trend was observed and further studies with large number of samples are worth considering in future studies.

\section{Conclusion}

DENV serotyping in Chittagong, Bangladesh clearly showed that all four DENV serotypes co-circulated during the last year (2019) dengue outbreak. Furthermore, 24\% of the infected populations suffered from concurrent multiple DENV infections. Perhaps, this is for the first time in Bangladesh where we report DENV4 as the most prevalent serotype, contrary to what have been reported previously (Shirin et al., 2019). Furthermore, DENV1 was the most prevalent serotype (present in $90 \%$ cases of multiple DENV infections) in concurrent multiple DENV infections. Such observations clearly suggested that DENV seroprevalence may be changing depending on geolocations from year to year. Moreover, the high occurrence of concurrent multiple DENV infections might be the causes of severe dengue in Bangladesh in recent years. Investigation of clinical sign-symptoms and blood cell counts clearly indicated that DENV3 infected persons had lower platelet counts and slow platelet replenishing trend which might also contribute to dengue disease severity associated with DENV3 infections. Inclusions of more samples are worth considering to explore rationale of dengue disease severity and clinical manifestation of dengue in future studies.

\section{Declarations}

\section{Ethical approval and consent of the participants}

Ethical approval was taken ethical review of committee of Chittagong Medical College and Hospital, Chittagong Bangladesh and written consent of the participants were taken. Availability of data and materials

Data sharing is not applicable as no data sets were generated or analysed during the current study.

\section{Acknowledgements}

MMI thanks Chittagong Medical College Hospital authority for ethical approval and providing samples.

\section{References}

1. Acosta, P.O.A, Granja, F, Meneses, C.A, Nascimento, I .A.S, Sousa, D.D, Lima, JÚNIOR W.P, Naveca, F.G. (2014), "False-Negative Dengue Cases in Roraima, Brazil: an Approach Regarding the High Number of Negative Results by Ns1 Ag Kits", Rev Inst Med Trop Sao Paulo, Vol. 56 No5,pp. 447-50.

2. Bhatt, S, Gething, P.W, Brady, O.J , Messina, J.P, Farlow, A.W, Moyes, C.L, Drake, J.M, Brownstein, J.S, Hoen, A.G, Sankoh, O, Myers, M.F, George, D.B, Jaenisch, T, William Wint, G.R, Simmons, C.P, Scott, T.W, Farrar, J.J, Hay, S.I (2013), "The global distribution and burden of dengue", Nature, Vol 496 pp 504-7.

3. Chastel, C. (2012), "Eventual role of asymptomatic cases of dengue for the introduction and spread of dengue viruses in non-endemic regions", Front. Physiol, Vol. 3 pp.70.

4. Crooks, A.J, Lee, J.M, Dowsett, A.B, Stephenson, J.R. (1990), "Purification and analysis of infectious virions and native non-structural antigens from cells infected with tick-borne encephalitis virus", Journal of Chromatography, Vol. 502 No.1,pp.59-68.

5. Dhanoa, A, Hassan, S.S, Ngim, C.F, Lau, C.F, Chan, T.S, Adnan, N.A.A, Eng, W.W.H, Gan, H.M, Rajasekaram, G. (2016), "Impact of dengue virus (DENV) co-infection on clinical manifestations, disease severity and laboratory parameters", BMC Infectious Diseases, Vol .16 No1,pp.406.

6. Duong, V, Lambrechts, L , Paul, R.E, Ly, S, Lay, R.S, Long, K.C, Huy, R, Tarantola, A, Scott, T.W, Sakuntabhai, A, Buchy, P. (2015), "Asymptomatic humans transmit dengue virus to mosquitoes", Proc Natl Acad Sci U S A, Vol.12 No.47, pp.14688-93.

7. Gubler, D.J. (1998), "Dengue and dengue hemorrhagic fever" Clin Microbiol Rev, Vol.11 No.3,pp.480-96.

8. Guzman, M.G, Alvarez, M, Halstead, S.B. (2013), "Secondary infection as a risk factor for dengue hemorrhagic fever/dengue shock syndrome: An historical perspective and role of antibody-dependent enhancement of infection", Arch. Virol, Vol.158 No.7,pp.1445-59.

9. Guzman, M.G, Halstead, S.B, Artsob, H , Buchy, P, Farrar, J, Gubler, D.J, Hunsperger, E, Kroeger, A, Margolis, H.S, Martí-nez, E,Nathan,M.B, Pelegrino, J.L, Simmons, C, Yoksan, S, Peeling, R.W. (2010), "Dengue: A continuing global threat", Nat Rev Microbiol, Vol.8 pp S7-S16.

10. Halstead, S.B (1966), "Mosquito-borne hemorrhagic fevers of South and Southeast Asia and the Western Pacific" Bull World Health Organ, Vol.35 No 1, pp.17-33

11. Innis, B.L, Nisalak, A, Nimmannitya, S, Kusalerdchariya, S, Chongswasdi, V, Suntayakorn, S, Puttisri, P, Hoke, C.H. (1989), "An enzyme -linked immunosorbent assay to characterize dengue infections where dengue and Japanese encephalitis co-circulate", Am J Trop Med Hyg, Vol.40 No.4,pp 418-27.

12. Kuno, G, Gómez, I, Gubler, D.J. (1991), “An ELISA procedure for the diagnosis of dengue infections", Journal of Virological Methods, Vol.33 No.1-2,pp.101-13.

13. Lanciotti, R.S, Calisher, C.H, Gubler, D.J, Chang, G.J, Vorndam, A.V. (1992), "Rapid detection and typing of dengue viruses from clinical samples by using reverse 
transcriptase-polymerase chain reaction", Journal of Clinical Microbiology, Vol.30 No.3, pp. 545-51.

14. Masyeni, S, Yohan, B, Sasmono, R.T. (2019), "Concurrent infections of dengue virus serotypes in Bali, Indonesia", BMC Res Notes, Vol. 12 No.1,pp.129.

15. Matheus, S, Deparis, X, Labeau, B, Lelarge, J, Morvan, J, Dussart, P. (2005), "Use of four dengue virus antigens for determination of dengue immune status by enzyme-linked immunosorbent assay of immunoglobulin G avidity", Journal of Clinical Microbiology, Vol.43 No.11, pp.5784-6.

16. Mehta, T.K, Shah, P.D. (2018), "Identification of prevalent dengue serotypes by reverse transcriptase polymerase chain reaction and correlation with severity of dengue as per the recent World Health Organization classification (2009)", Indian Journal of Medical Microbiology, Vol. 36 No.2, pp.273-8.

17. Minnie, D, Srinivasan, S. (2012), "Preprocessing and generation of association rules for automated blood cell counter data in haematology. 2012 International Conference on Recent Advances in computing and software system, pp.27-32.

18. Mollah AH, Nahar N, Step on ta Paediatrics. $5^{\text {th }}$ Edition 2020. Fever and Rash. Ch 15, P 154-65.

19. Nimmannitya, S, Thisyakorn, U, Hemsrichart, V. (1987), "Dengue haemorrhagic fever with unusual manifestations" Southeast Asian J. Trop. Med. Public Health,Vol. 18 No.3,pp.398-406. No. 117. Geneva. 2015", World Health Organization.

20. Pok, K.Y, Lai, Y.L, Sng, J, Ng, L.C. (2010), "Evaluation of nonstructural 1 antigen assays for the diagnosis and surveillance of dengue in Singapore", Vector Borne and Zoonotic Diseases, Vol. 10 No.10,pp.1009-16.

21. Reddy, M.N, Dungdung,R, Valliyott, L, Pilankatta, R. (2017), "Occurrence of concurrent infections with multiple serotypes of dengue viruses during 2013-2015 in northern Kerala, India", Peer J, Vol.5.pp.e2970.

22. Senaratne, U.T.N, Murugananthan, K, Sirisena ,P.D.N.N, Carr J.M, Noordeen F. (2020), "Dengue virus coinfections with multiple serotypes do not result in a different clinical outcome compared to mono-infections", Epidemiol Infect, Vol.148 pp.119.

23. Shirin, T, Muraduzzaman, A.K.M, Alam, A.N, Sultana, S, Siddiqua, M, Khan, M.H, Akram, A, Sharif, A.R, Hossain,S, Flora, M.S. (2019), "Largest dengue outbreak of the decade with high fatality may be due to reemergence of DEN-3 serotype in Dhaka, Bangladesh, necessitating immediate public health attention", New Microbes New Infect, Vol.29.pp.100511.

24. Singh, A, Bisht, P, Bhattacharya, S, Guchhait, P. (2020), "Role of Platelet Cytokines in Dengue Virus Infection", Front Cell Infect Microbiol, Vol.10 pp. 561366.

25. Soo, K.M, Khalid, B, Ching, S.M, Chee, H.Y(2016), "Meta-analysis of dengue severity during infection by different dengue virus serotypes in primary and secondary infections" PLoS One, Vol.11 No 5,pp.e0154760.

26. Stanaway, J.D, Shepard, D.S, Undurraga, E.A, Halasa, Y.A, Coffeng, L.E, Brady, O.J, Hay, S.I, Bedi, N, Bensenor, I.M , Castañeda-Orjuela, C.A, Chuang, T.W, Gibney, K.B, Memish, Z.A, Rafay, A, Ukwaja, K.N, Yonemoto, N, Murray, C.J.L. (2016), "The global burden of dengue: an analysis from the Global Burden of Disease Study 2013". Lancet Infect Dis, Vol.16 No.6, pp .712-23.

27. Tazeen, A, Afreen ,N, Abdullah, M, Deeba, F, Haider, S.H, Kazim, S.N, Ali, S, Naqvi,H, Broor, S, Ahmed, A, Parveen S. (2017), "Occurrence of co-infection with dengue viruses during 2014 in New Delhi, India", Epidemiology and Infection, Vol .145 No.1, pp.67-77.

28. Ten Bosch, Q.A, Clapham, H.E, Lambrechts, L, Duong, V, Buchy, P, Althouse, B.M, Lloyd, A.L, Waller, L.A, Morrison, A.C, Kitron, U, Vazquez-Prokopec, G.M, Scott, T.W, Perkins, T.A. (2018), "Contributions from the silent majority dominate dengue virus transmission", PLoS Pathog, Vol. 14 No.5, pp.e1006965.

29. Thomas, L, Verlaeten, O, Cabié, A, Kaidomar, S, Moravie, V,Martial, J, Najioullah, F, Plumelle,Y, Fonteau, C, Dussart, P, Césaire, R. (2008), "Influence of the dengue serotype, previous dengue infection and plasma viral load on clinical presentation and outcome during a dengue- 2 and dengue-4 co-epidemic", American Journal of Tropical Medicine and Hygiene, Vol.78 No.6,pp.990-998.

30. Utama, I.M.S, Lukman, N, Sukmawati, D.D, Alisjahbana, B, Alam, A, Murniati, D, Utama, M.G.D.L, Puspitasari, D, Kosasih, H, Laksono, I, Karyana, M, Karyanti, M.R, Hapsari, M.M.D.E.A.H, Meutia, N, Liang, C.J, Wulan, W.N, Lau, C.Y, Parwati ,K.T.M.P. (2019), "Dengue viral infection in Indonesia: Epidemiology, diagnostic challenges, and mutations from an observational cohort study”, PLoS Negl Trop Dis, Vol.13No.10,pp.e0007785.

31. World Health Organization (WHO), WHO, (2016) "Dengue and severe dengue. Fact sheet

32. World Health Organization. National Malaria Elimination \& Aedes Transmitted Disease Control Program Diseases Control Unit. National Guideline for Clinical Management of Dengue Syndrome. $4^{\text {Th }}$ Edition 2018. Islam QT, Amin R, Bitu MR et al. Lab investigation for Dengue Diagnosis and Management Ch 3.P.18

33. Yong, Y.K, Thayan, R, Chong, H.T, Tan, C.T, Sekaran, S.D. (2007), "Rapid detection and serotyping of dengue virus by multiplex RT-PCR and real-time SYBR green RT-PCR”, Singapore Med J, Vol.48 No.7,pp. 662-8. 